\title{
ON GROUP GRADED RINGS SATISFYING POLYNOMIAL IDENTITIES
}

\author{
by A. V. KELAREV and J. OKNIŃSKI
}

(Received 2 November, 1993)

A number of classical theorems of ring theory deal with nilness and nilpotency of the Jacobson radical of various ring constructions (see [10], [18]). Several interesting results of this sort have appeared in the literature recently. In particular, it was proved in [1] that the Jacobson radical of every finitely generated PI-ring is nilpotent. For every commutative semigroup ring $R S$, it was shown in [11] that if $J(R)$ is nil then $J(R S)$ is nil. This result was generalized to all semigroup algebras satisfying polynomial identities in [15] (see [16, Chapter 21]). Further, it was proved in [12] that, for every normal band $B$, if $J(R)$ is nilpotent, then $J(R B)$ is nilpotent. A similar result for special band-graded rings was established in [13, Section 6]. Analogous theorems concerning nilpotency and local nilpotency were proved in [2] for rings graded by finite and locally finite semigroups.

This paper is devoted to the radicals of group graded rings, which have been actively investigated by many authors (see [10], [14]). Let $G$ be a group. An associative ring $R=\bigoplus_{g \in G} R_{g}$ is said to be $G$-graded (strongly $G$-graded) if $R_{g} R_{h} \subseteq R_{g h}$ (respectively, $R_{g} R_{h}=R_{g h}$ ) for all $g, h \in G$.

First, we consider algebras over a field of characteristic zero. In this case our result will be also of interest in connection with the well-known problem of finding necessary and sufficient conditions for the Jacobson radical to be homogeneous. An ideal $I$ of $R=\bigoplus_{g \in G} R_{g}$ is said to be homogeneous if $I=\bigoplus_{g \in G} I \cap R_{g}$. This problem has not been solved even for u.p.-groups (see [7], [8], [10]). Polynomial identities give what appears to be the first natural sufficient condition which is applicable to the case of arbitrary groups.

THEOREM 1. Let $G$ be a group with identity $e$, and let $R=\bigoplus_{g \in G} R_{g}$ be a $G$-graded $P I$-algebra over a field of characteristic zero. If the Jacobson radical $J\left(R_{e}\right)$ is nil, then $J(R)$ is a homogeneous nil ideal of $R$.

The following corollary to the main theorem is worth mentioning.

COROllary 2. Let $G$ be a group with identity $e$, and let $R=\bigoplus_{g \in G} R_{g}$ be a strongly $G$-graded PI-algebra over a field of characteristic zero. If $J\left(R_{e}\right)$ is nilpotent, then $J(R)$ is nilpotent.

It is impossible to replace strongly graded algebras by ordinary group graded algebras in Corollary 2. Indeed, let $A$ be the free commutative algebra with free generators $a_{1}, a_{2}, \ldots$ Denote by $I$ the ideal of $A$ generated by $a_{1}, a_{2}^{2}, a_{3}^{3}, \ldots$ Then $A / I$ is positively graded, and so $A / I=\bigoplus_{z \in Z} A_{z}$ where $Z$ is the infinite cyclic group. Although $A_{0}=0$, it is clear that $J(A / I)=A / I$ is not nilpotent.

One cannot omit the restriction on the characteristic of the field neither in Theorem

Glasgow Math. J. 37 (1995) 205-210. 
1 nor in Corollary 2, as the results on the Jacobson radical of group algebras show (see [10, Section 44]). However, the conclusion of Theorem 1 concerning nilness holds for arbitrary PI-rings. Namely, the following graded analogue of the celebrated Braun's Nullstellensatz for PI-rings (see [1], [18]) is valid.

THEOREM 3. Let $G$ be a group with identity e, and let $R=\bigoplus_{g \in G} R_{g}$ be a $G$-graded
-ring. If $J\left(R_{e}\right)$ is nil, then $J(R)$ is nil, too. PI-ring. If $J\left(R_{e}\right)$ is nil, then $J(R)$ is nil, too.

Throughout $R=\bigoplus_{g \in G} R_{g}$ will be a $G$-graded ring, $G$ a group with identity $e$. Let $T$ be a subset of $G$. Put $R_{T}=\bigoplus_{t \in T} R_{r}$. For any $r \in R$, say $r=\sum_{g \in G} r_{g}$ where $r_{g} \in R_{g}$, we put $r_{T}=\sum_{t \in T} r_{t}$. If $I \subseteq R$ and $g \in G$, then we put $I_{g}=I \cap R_{g}$ and $I_{T}=I \cap R_{T}$. Further, for $r \neq 0$, denote the set of all non-zero homogeneous summands $r_{g}$ of $r$ by $H(r)$, and put $\operatorname{supp}(r)=\left\{g \in G \mid r_{g} \neq 0\right\}$. Put $H(0)=0, \operatorname{supp}(0)=\varnothing$. Clearly, $H(r)$ and $\operatorname{supp}(r)$ are finite sets. Let $H(I)=\bigcup_{r \in I} H(r)$. Then $H(R)$ is the set of all homogeneous elements of $R$.

Evidently, $H(R)$ is a multiplicative subsemigroup of $R$. By the length of $r$ we mean $|\operatorname{supp}(r)|$. Recall that a semigroup $S$ is said to be permutational if there exists $n>1$ such that, for any $n$ elements $x_{1}, \ldots, x_{n}$ of $S$, their product can be rearranged as $x_{1} \ldots x_{n}=$ $x_{\sigma 1} \ldots x_{\sigma n}$ for a non-trivial permutation $\sigma$. Every PI-ring (or PI-algebra) satisfies a multilinear identity, i.e. an identity of the form

$$
x_{1} \ldots x_{n}+\sum_{1 \neq \sigma \in S_{n}} k_{\sigma} x_{\sigma 1} \ldots x_{\sigma n}=0
$$

where $S_{n}$ is the symmetric group, $k_{\sigma}$ are integers (elements of the field in the case of algebras, see [17]). Let us begin with a known lemma (see [10, Proposition 6.18]), which will be used repeatedly.

LEMMA 4. Let $R$ be a $G$-graded ring, $H$ a subgroup of $G$. Then $J\left(R_{H}\right) \supseteq R_{H} \cap J(R)$.

Lemma 5. Let $R$ be a G-graded PI-ring, $I$ a homogeneous ideal of $R$ contained in $J(R)$. If $I_{e}$ is nil, then I is nil.

Proof. Take any element $r$ in $H(I)$. Since $I$ is homogeneous, $r \in I$. Let $r \in J(R) \cap R_{g}$, where $g \in G$. If $g$ is a periodic element, then there exists a positive integer $n$ such that $r^{n} \in I \cap R_{e}=I_{e}$, and so $r$ is nilpotent. Further, assume that $g$ is not periodic. Denote by $T$ the infinite cyclic group generated in $G$ by $g$. Lemma 4 shows that $r \in J\left(R_{T}\right)$, and therefore $r$ is nilpotent again in view of [10, Theorem 32.5]. Thus $H(I)$ is a multiplicative nil subsemigroup of $R$. Since $R$ satisfies a polynomial identity, it follows from [17, Theorem 1.6.36], that $I$ is nil, as required.

Lemma 6. Let $G$ be a permutational group, $R$ a $G$-graded PI-ring. If $J\left(R_{e}\right)$ is nil, then $J(R)$ is nil.

Proof. By [16, Theorem 19.8], $G$ is finite-by-abelian-by-finite. Take any $r \in J\left(R_{G}\right)$. Denote by $S$ the subgroup generated in $G$ by the support of $r$. It is easily seen that $S$ is also finite-by-abelian-by-finite. Lemma 4 implies $r \in J\left(R_{S}\right)$, and so without loss of 
generality we may assume that $G$ is finitely generated itself. Then assertion (2.2) of [4] tells us that $G$ is abelian-by-finite, i.e. $G$ has an abelian normal subgroup $A$ of finite index. Then $A$ is finitely generated, too (see [9]). Therefore $G$ contains a torsion-free abelian subgroup $T$ of finite index. By [10, Corollary 22.8], $R$ is graded by the finite group $G / T$ with the identity component $R_{T}$. Therefore [15, Lemma 1.1(1)], shows that it suffices to prove that $J\left(R_{T}\right)$ is nil. However, $J\left(R_{T}\right)$ is homogeneous by [10, Theorem 30.28], because $T$ is torsion-free abelian. Lemma 5 completes the proof.

Lemma 7. Let $G$ be a permutational group, $R$ a $G$-graded PI-algebra over a field of characteristic zero. If $J\left(R_{e}\right)$ is nil, then $J(R)$ is homogeneous.

Proof. We shall verify that $H(J(R))$ consists of nilpotent elements. Then [17, Theorem 1.6.36], will show that $H(J(R))$ generates a homogeneous nil ideal $I$ in $R$, and so $J(R)=I$ is homogeneous.

Pick any $r \in J(R)$ and $g \in \operatorname{supp}(r)$. We claim that $r_{g}$ is nilpotent. As in the beginning of the proof of Lemma 6, we may assume that $G$ has a torsion-free abelian subgroup $T$ of finite index. If we look at the natural $G / T$-gradation of $R$ and apply [10, Theorem 30.28 (b)], and the fact that our field has characteristic zero, then we conclude that $J(R)$ is $G / T$-homogeneous. We may assume that the whole $\operatorname{supp}(r)$ is contained in one $T$-coset of $G$ (otherwise we would pass to the $G / T$-homogeneous summand of $r$ corresponding to the coset containing $g$ ). Since $G / T$ is finite, there exists a positive integer $n$ such that $r r_{g}^{n} \in R_{T}$. Given that $J\left(R_{e}\right)$ is nil, [10, Theorem 32.5], implies that all the homogeneous summands of $r r_{g}^{n}$ are nilpotent. Therefore $r_{g}$ is nilpotent, as required.

Proof of Theorem 1. By Lemma 5 the largest homogeneous ideal $I$ of $R$ contained in $J(R)$ is nil. Obviously, $R / I$ is a $G$-graded ring, and $J(R / I)=J(R) / I$. Therefore it suffices to prove Theorem 1 for $R / I$. To simplify the notation we may assume that from the very beginning $I=0$.

Suppose that $J(R) \neq 0$. Choose a non-zero element $r$ with a minimal length in $J(R)$. Denote by $T$ and $S$ the subgroup and, respectively, subsemigroup generated in $G$ by $\operatorname{supp}(r)$. Let $M=M(r)$ be the multiplicative subsemigroup generated in $R$ by $H(r)$. We claim that $H(r)$ consists of nilpotent elements.

If $S$ is permutational, then the group $T$ is permutational too, by [16, Theorem 19.8], and so all elements in $H(r)$ are nilpotent in view of Lemmas 6 and 7.

Further, consider the case where $S$ is not permutational. Let $n$ be the degree of a multilinear identity (1) of $R$. There exist elements $s_{1}, \ldots, s_{n}$ in $S$ such that $s_{1} \ldots s_{n} \neq$ $s_{\sigma 1} \ldots s_{\sigma n}$ for all $\sigma \in S_{n}$ such that $\sigma \neq 1$. Clearly, there exist $x_{1}, \ldots, x_{n} \in M$ such that $x_{i} \in R_{s_{i}}$ for all $i=1, \ldots, n$. Applying (1) to the elements $x_{1}, \ldots, x_{n}$ we get

$$
x_{1} \ldots x_{n} \in R_{s_{1} \ldots s_{n}} \cap \sum_{1 \neq \sigma \in S_{n}} R_{s_{\sigma 1} \ldots s_{o n}}
$$

whence $x_{1} \ldots x_{n}=0$. It follows that $y_{1} \ldots y_{m}=0$ for some $y_{1}, \ldots, y_{m} \in H(r)$. Then we can choose $m$ and the $y_{1}, \ldots, y_{m}$ such that $y_{1} \ldots y_{m-1} \neq 0$. Now look at the product $y_{1} \ldots y_{m-1} r$. It also belongs to $J(R)$ but has fewer homogeneous summands than $r$. Hence $y_{1} \ldots y_{m-1} r=0$ by the choice of $r$. Since $G$ is a group, we get $y_{1} \ldots y_{m-1} H(r)=0$. Further, we can look at $y_{1} \ldots y_{m-2} r H(r)=0$ and infer $y_{1} \ldots y_{m-2}\left(H(r)^{2}\right)=0$. Reasoning like this $m$ times, we conclude $(H(r))^{m}=0$. In particular, all elements in $H(r)$ are nilpotent, again. 
Denote by $L$ the ideal generated in $H(R)$ by $H(r)$. Each non-zero element of $L$ is a homogeneous summand of a certain element of positive minimal length in $J(R)$. It follows from what we have proved that $L$ is a nil ideal of $H(R)$. Hence $L$ is locally nilpotent by [17, Theorem 1.6.36]. Therefore $L$ generates a nil subalgebra $K$ in $R$. Evidently, $K$ is a homogeneous ideal of $R$, and so $K \subseteq I$, contradicting the fact that $I=0$. Thus $J(R)=I$, and so $J(R)$ is a homogeneous nil ideal of $R$.

Proof of Corollary 2. By Theorem 1 we get $J(R)=\bigoplus_{g \in G} I_{g}$, where $I_{g}=I \cap R_{g}$. Denote by $n$ the nilpotency index of $J\left(R_{e}\right)$. Lemma 4 implies $I_{e} \subseteq J\left(R_{e}\right)$, and so $\left(I_{e}\right)^{n}=0$. We claim that $J(R)^{2 n}=0$.

To this end we need only to verify that $x_{1} \ldots x_{2 n}=0$ for arbitrary homogeneous elements $x_{1}, \ldots, x_{2 n} \in J(R)$. Let $x_{i} \in I_{g_{i}}$, where $i=1, \ldots, 2 n$. Given that $x_{2} \in R_{g_{2}}=$ $R_{g_{1}^{-1}} R_{g_{1 g_{2}}}$, we can find $y_{j_{1}}^{(1)} \in R_{g_{1}^{-1}}$ and $z_{j_{1}}^{(1)} \in R_{g_{1} g_{2}}$ such that $x_{2}=\sum_{j_{1}} y_{j_{1}}^{(1)} z_{j_{1}}^{(1)}$. Then $x_{1} y_{j_{1}}^{(1)} \in I_{e}$ for all $j_{1}$. Further, suppose that for some $i=2, \ldots, n$ elements $y_{j_{i-1}}^{(i-1)}$ and $z_{j_{i-1}}^{(i-1)}$ have been introduced such that $x_{2 i-2}=\sum_{j_{i-1}} y_{j_{i-1}}^{(i-1)} z_{j-1}^{(i-1)}$ and all $z_{j_{i-1}}^{(i-1)} \in R_{g_{1} \ldots g_{2 i-2}}$. Then $x_{2 i} \in R_{g_{2 i}}=$ $R_{\left(g_{1} \ldots g_{2-1}\right)^{-1}} R_{g_{1} \ldots g_{2}}$ and so there exist homogeneous elements $y_{j_{i}}^{(i)} \in R_{\left(g_{1} \ldots g_{2-1}\right)^{-1}}$ and $z_{j_{i}}^{(i)} \in$ $R_{g_{1} \ldots g_{2 i}}$ such that $x_{2 i}=\sum_{j_{i}} y_{j_{i}}^{(i)} z_{j_{i}}^{(i)}$ and all $z_{j_{i-1}}^{(i-1)} x_{2 i-1} y_{j_{i}}^{(i)}$ belong to $I_{e}$. Therefore

$$
\begin{aligned}
x_{1} \ldots x_{2 n} & =\sum_{j_{1}, \ldots j_{n}}\left(x_{1} y_{j_{1}}^{(1)}\right)\left(z_{j_{1}}^{(1)} x_{3} y_{j_{2}}^{(2)}\right)\left(z_{j_{2}}^{(2)} x_{5} y_{j_{3}}^{(3)}\right) \ldots\left(z_{j_{n-1}}^{(n-1)} x_{2 n-1} y_{j_{n}}^{(n)}\right) z_{j_{n}}^{(n)} \\
& \in I_{e}^{n} \sum_{j_{n}} z_{j_{n}}^{(n)}=0
\end{aligned}
$$

which completes the proof.

Proof of Theorem 3. We shall prove that $J(R)=B(R)$, where $B(R)$ denotes the Baer radical of $R$. To this end we show that $R / B(R)$ is semisimple. Suppose to the contrary that $J(R / B(R)) \neq 0$. Since $R / B(R)$ is a subdirect product of prime PI-rings, there exists a prime PI-ring $\bar{R}$ and a homomorphism $f$ of $R$ onto $\bar{R}$ such that $f(J(R)) \neq 0$. By Posner's theorem (see [17]) $\bar{R}$ is contained in a matrix ring $D_{m}$, where $D$ is a division ring. For any $r \in R$ and $T \subseteq R$, put $\bar{r}=f(r), \bar{T}=f(T)$.

Consider the set $L$ of all $x \in H(R)$ such that either $\bar{x}=0$ or $\bar{x}$ has the smallest non-zero rank in $D_{m}$. By [16, Theorem 1.6], all non-zero elements of $\bar{L}$ lie in the same completely 0 -simple factor $F$ of the multiplicative semigroup $D_{m}$. Obviously, $\bar{L}$ is a multiplicative ideal of $\overline{H(R)}$, and so $L$ is a multiplicative ideal of $H(R)$.

Put $M=L J(R) L=\{x a y \mid a \in J(R), x, y \in L\}$. Clearly, $M$ is a multiplicative subsemigroup of $R$, because $J(R)$ is an ideal of $R$. Denote by $I$ the subring generated in $R$ by $L$. It follows that $I$ is an ideal of $R$ and $\bar{I}$ is an ideal of $\bar{R}$. Since $\bar{I} \neq 0$ and $\bar{R}$ is prime, we get $\overline{I J(R)} \neq 0$, and so $\overline{I J(R) I} \neq 0$. Since $I$ consists of all finite sums of elements of $L$, we get $\bar{M} \neq 0$. We shall show that every element in $\bar{M}$ is nilpotent.

Fix any non-zero $\bar{w} \in \bar{M}$. There exists $w=a x b$, such that $a, b \in L, x \in J(R)$, $x=\sum_{k=1}^{s} x_{k}$, and all $x_{k}$ are homogeneous. Elements $\bar{a}$ and $\bar{b}$ belong to the same completely 0 -simple factor $F$ of $D_{m}$. By [16, Theorem 1.3], we can represent $F$ as a Rees 
matrix semigroup $F=\mathcal{M}\left(G^{0} ; I, \Lambda ; P\right)$. (The definitions and standard properties of completely 0 -simple semigroups and Rees matrix semigroups can be also found in each of the following monographs [3], [5], [6].) Let $\bar{a}=(i, g, \lambda)$ and $\bar{b}=(j, h, \mu)$, where $i, j \in I$, $\lambda, \mu \in \Lambda, g, h \in G$. It is routine to verify that $\overline{a x_{k}} \bar{b} \in\left(i, G^{0}, \mu\right)$ for every $k=1, \ldots, s$.

If $p_{\mu i}=0$, then $\left(i, G^{0}, \mu\right)^{2}=0$ in $F$, and therefore $(a x \bar{b})^{2}=0$ in $F$. Hence every element $\left(\overline{a x_{k}} \bar{b}\right)\left(\overline{a x_{l}} \bar{b}\right)$ has a smaller rank in $D_{m}$ than $\bar{a}$. Therefore $\left(\overline{a x_{k}} \bar{b}\right)\left(\overline{a x_{l}} \bar{b}\right)=0$ where $1 \leq k, l \leq s$. Thus $\bar{w}^{2}=0$, as required.

Further, consider the case where $p_{\mu i} \neq 0$. By [16, Lemma 1.4], $P=(i, G, \mu)$ is a multiplicative subgroup of $D_{m}$. Put $T=\left\{g \in G \mid \bar{R}_{g} \cap P \neq 0\right\}$. Clearly, $T$ is a subsemigroup of $G$. Let $n$ be the degree of a multilinear identity (1) satisfied in $R$. For any $g_{1}, \ldots, g_{n} \in T$, we can choose $r_{k} \in R_{g_{k}}$ such that $\overline{r_{k}} \in P$, where $k=1, \ldots, n$. Applying (1) we get (2), which implies that $T$ is permutational. By [16, Theorem 19.8], $T$ generates a permutational subgroup $Q$ in $G$. Lemma 6 shows that $J\left(R_{Q}\right)$ is nil.

Since all non-zero summands $\overline{a x_{k}} \bar{b}$ belong to $P$, where $k=1, \ldots, s$, we get $a x_{k} b \in R_{Q}$, and so $a x b \in J\left(R_{Q}\right)$ by Lemma 4 . Therefore $w=a x b$ is a nilpotent element.

Thus $\bar{M}$ is a multiplicative nil subsemigroup of $D_{m}$. Hence $\bar{M}^{q}=0$ for some $q>1$ by [18, Proposition 2.6.30]. Since $M$ is, evidently, closed under multiplication by elements of $H(R)$, it follows that $\bar{M}$ generates a nilpotent ideal in $\bar{R}$. This contradicts the primeness of $\bar{R}$ and completes the proof.

\section{REFERENCES}

1. A. Braun, The nilpotency of the radical in a finitely generated PI-ring, J. Algebra 89 (1984), 375-396.

2. M. V. Clase and E. Jespers, On the Jacobson radical of semigroup graded rings, J. Algebra 169 (1994), 79-97.

3. A. H. Clifford and G. B. Preston, The algebraic theory of semigroups, vol. 1 (American Mathematical Society, 1961).

4. M. Curzio, P. Longobardi, M. Maj and D. J. S. Robinson, A permutational property of groups, Arch. Math. (Basel) 44 (1985), 385-389.

5. P. M. Higgins, Techniques of semigroup theory (Oxford University Press, 1992).

6. J. M. Howie, An introduction to semigroup theory, London Mathematical Society Monographs No. 7 (Academic Press, 1976).

7. E. Jespers, Radicals of graded rings, Theory of radicals, Szekszárd, 1991, Colloq. Math. Soc. János Bolyai 61 (North Holland, 1993), 109-130.

8. E. Jespers, J. Krempa and E. R. Puczyłowski, On radicals of graded rings, Comm. Algebra 10 (1982), 1849-1854. 1979).

9. M. I. Kargapolov and Ju. I. Merzljakov, Fundamentals of the Theory of Groups (Springer,

10. G. Karpilovsky, The Jacobson radical of classical rings, Pitman Monographs (Longman/John Wiley, 1991).

11. W. D. Munn, The algebra of a commutative semigroup over a commutative ring with unity, Proc. Roy. Soc. Edinburgh Sect. A 99 (1985), 387-398.

12. W. D. Munn, The Jacobson radical of a band ring, Math. Proc. Cambridge Philos. Soc. 105 (1989), 277-283.

13. W. D. Munn, A class of band-graded rings, J. London Math. Soc. 45 (1992), 1-16.

14. C. Năstăsescu and F. Van Oystaeyen, Graded ring theory (North Holland, 1982).

15. J. Okniniski, On the radical of semigroup algebras satisfying polynomial identities, Math. Proc. Cambridge Philos. Soc. 99. (1986), 45-50. 
16. J. Okniński, Semigroup algebras (Marcel Dekker, 1991).

17. L. H. Rowen, Polynomial identities in ring theory (Academic Press, 1980).

18. L. H. Rowen, Ring theory (Academic Press, 1988).

Department of Mathematics

UNIVERSITY OF TASMANIA

HOBART, GPO BoX 252C

TASMANIA 7001

Australia
Department of Mathematics

UNIVERSITY OF WARSAW

BANACHA 2

02-097 WARSAW

Poland 